\title{
Phylogenetic classification of Escherichia coli O 157:H7 strains of human and bovine origin using a novel set of nucleotide polymorphisms
}

\author{
Michael L Clawson ${ }^{\natural *}$, James E Keen ${ }^{\sharp *}$, Timothy PL Smith*, Lisa M Durso*, \\ Tara G McDaneld ${ }^{*}$, Robert E Mandrell ${ }^{\dagger}$, Margaret A Davis ${ }^{\ddagger}$ and \\ James L Bono*
}

Addresses: *United States Department of Agriculture (USDA), Agricultural Research Service (ARS), US Meat Animal Research Center (USMARC), State Spur 18D, Clay Center, NE 68933, USA. 'USDA, ARS, Western Regional Research Center, Buchanan St, Albany, CA 94710, USA. "Washington State University, Department of Pathology, Bustad Hall, Pullman, WA 99164-7040, USA. \$Current address: University of Nebraska, Great Plains Veterinary Educational Center, Clay Center, NE 68933, USA.

$\ltimes$ These authors contributed equally to this work.

Correspondence: James L Bono. Email: jim.bono@ars.usda.gov

Published: 22 May 2009

Genome Biology 2009, 10:R56 (doi:10.1 186/gb-2009-10-5-r56)

The electronic version of this article is the complete one and can be found online at http://genomebiology.com/2009//0/5/R56
Received: 21 January 2009

Revised: 20 March 2009

Accepted: 22 May 2009

(c) 2009 Clawson et al.; licensee BioMed Central Ltd.

This is an open access article distributed under the terms of the Creative Commons Attribution License (http://creativecommons.org/licenses/by/2.0), which permits unrestricted use, distribution, and reproduction in any medium, provided the original work is properly cited.

\begin{abstract}
Background: Cattle are a reservoir of Shiga toxin-producing Escherichia coli OI57:H7 (STEC OI57), and are known to harbor subtypes not typically found in clinically ill humans. Consequently, nucleotide polymorphisms previously discovered via strains originating from human outbreaks may be restricted in their ability to distinguish STEC OI57 genetic subtypes present in cattle. The objectives of this study were firstly to identify nucleotide polymorphisms in a diverse sampling of human and bovine STEC OI57 strains, secondly to classify strains of either bovine or human origin by polymorphism-derived genotypes, and finally to compare the genotype diversity with pulsedfield gel electrophoresis (PFGE), a method currently used for assessing STEC OI57 diversity.

Results: High-throughput 454 sequencing of pooled STEC OI 57 strain DNAs from human clinical cases $(n=91)$ and cattle $(n=102)$ identified 16,218 putative polymorphisms. From those, 178 were selected primarily within genomic regions conserved across $E$. coli serotypes and genotyped in 261 STEC OI57 strains. Forty-two unique genotypes were observed that are tagged by a minimal set of 32 polymorphisms. Phylogenetic trees of the genotypes are divided into clades that represent strains of cattle origin, or cattle and human origin. Although PFGE diversity surpassed genotype diversity overall, ten PFGE patterns each occurred with multiple strains having different genotypes.

Conclusions: Deep sequencing of pooled STEC O157 DNAs proved highly effective in polymorphism discovery. A polymorphism set has been identified that characterizes genetic diversity within STEC OI57 strains of bovine origin, and a subset observed in human strains. The set may complement current techniques used to classify strains implicated in disease outbreaks.
\end{abstract}




\section{Background}

Shiga toxin-producing Escherichia coli O157:H7 (STEC O157) recently emerged as a cause of diarrhea, hemorrhagic colitis, and hemolytic uremic syndrome (HUS) [1]. STEC O157 cause an estimated 73,480 illnesses each year in the United States [2] and probably evolved from a progenitor of E. coli $\mathrm{O}_{55}: \mathrm{H} 7$, a source of infantile diarrhea [3]. The STEC O157 5.5-Mb genome contains a 4.1-Mb backbone that is shared with $E$. coli K-12 and thought to be conserved across most $E$. coli serotypes [4,5]. Much of the remaining genome originates from horizontal transfer, with a significant contribution from bacteriophages $[4,5]$. The loss and gain of genes through horizontal transfer, coupled with nucleotide variation distributed throughout the STEC O157 genome, serve in both recording the evolution and defining the diversity of this pathogenic serotype [6-8].

Detection of genetic diversity between STEC O157 strains is an important component of outbreak investigations. Heterogeneity between STEC O157 strains has been detected through multilocus sequence tagging [9], octamer and PCRbased genome scanning [10,11], phage typing [12,13], multiple-locus variable-number tandem repeat analysis [14], microarrays [15,16], nucleotide polymorphism assays [8], phage integration patterns coupled with genome polymorphisms [17,18], and pulsed-field gel electrophoresis (PFGE) $[19,20]$. Of these, PFGE is currently the method of choice for distinguishing between STEC O157 strains implicated in outbreaks [21], and entails standardized chromosome digestions with $X b a \mathrm{I}$, and separation of DNA segments through gel electrophoresis[22]. Differing banding patterns are used to identify genetic diversity between STEC O157 strains. However, PFGE does not effectively show evolutionary descent between epidemiologically related strains $[8,23]$. Additionally, the standardized PFGE method is unreliable for determining genetic relatedness between STEC O157 strains that are epidemiologically unrelated [24].

Nucleotide polymorphisms, if sufficiently present within microbial populations, such as STEC O157, are highly amenable for determining genetic relatedness and descent between either epidemiologically related or unrelated strains [25]. Single nucleotide polymorphisms (SNPs) have been recently identified throughout the STEC O157 genome $[15,16,26]$ and some have been used to identify variation between STEC O157 strains originating from clinically ill humans [8]. Thirtynine SNP-based STEC O157 genotypes were identified that defined nine phylogenetic clades, of which one associated with increased hemolytic uremic syndrome, a serious complication of STEC O157 infection [8]. Thus, SNPs have been employed in the classification of STEC O157 by phylotype, and for distinguishing a subpopulation with increased human virulence.

Cattle are a reservoir of STEC O157 and harbor subtypes that are not typically observed in humans $[10,26]$. Consequently,
SNPs ascertained exclusively with strains associated with human outbreaks [16] may be ineffective at distinguishing a greater proportion of STEC O157 genetic diversity present in cattle. Given that strains of any genetic subtype may be drawn into a human outbreak investigation, and/or food recall, an ability to detect the fullest spectrum of STEC O157 genetic diversity with nucleotide polymorphisms would be useful in any STEC O157 investigation. Additionally, a greater understanding of STEC O157 genetic diversity, and how it relates to human pathogenesis, may lead to the identification of alleles that are directly involved with increased human virulence.

The main goal of this study was to sequence the genomes (1x) of 193 diverse STEC O157 strains and identify a set of nucleotide polymorphisms that classify STEC O157 of either bovine or human origin by genotype. Reported here are 42 unique polymorphism-derived STEC O157 genotypes that are tagged by a minimal set of 32 polymorphisms. Phylogenetic trees produced by the genotypes are split into clades that represent strains of cattle origin, or cattle and human origin. These results indicate that heterologous members of the STEC 0157 serotype are distinguishable through nucleotide polymorphisms, and support the notion that a subset of STEC O157 harbored in cattle causes the majority of human disease.

\section{Results \\ Sequencing coverage of 193 STEC OI57 strains}

Approximately $1 \times$ genome coverage of 193 STEC O157 strains was obtained through 454 GS FLX shotgun sequencing of three STEC O157 DNA pools (see Additional data file 1 for supplementary strain, PFGE, and genotype information). The DNA pools were designed to account for: host origin; and the alleles of a polymorphism in the translocated intimin receptor gene (tir $255 \mathrm{~T}>\mathrm{A}$ ), as STEC 0157 with the tir $255 \mathrm{~T}>\mathrm{A}$ A allele are rarely isolated from clinically ill humans [26]. A total of $1.306 \mathrm{~Gb}$ of genomic sequence was obtained from DNA pools of: 51 strains of bovine origin, tir $255 \mathrm{~T}>\mathrm{A}$ A allele $(346.2 \mathrm{Mb})$; 51 strains of bovine origin tir $255 \mathrm{~T}>\mathrm{A}$ T allele $(402.6 \mathrm{Mb})$; and 91 strains of human origin, of which all had the tir $255 \mathrm{~T}>\mathrm{A} \mathrm{T}$ allele $(557.5 \mathrm{Mb})$. Given that the STEC O157 genome is approximately $5.5 \mathrm{Mb}$, the depth of sequence obtained for each of the three pools averages to slightly more than $1 \times$ whole genome coverage for each of the 193 strains sequenced in this study.

\section{Polymorphism identification and validation}

A total of 16,218 putative nucleotide and/or insertion deletion polymorphisms were identified and mapped onto the Sakai STEC O157 reference genome with Roche GS Reference Mapper Software (Nutley, NJ, USA). Of these, 9,528 mapped to prophages integrated throughout $12.2 \%$ of the Sakai genome (Figure 1). Phage integration loci are problematical for both SNP discovery and validation, as multiple integrations within the STEC O157 genome have resulted in large stretches of paralogous sequence that are virtually indistinguishable from 


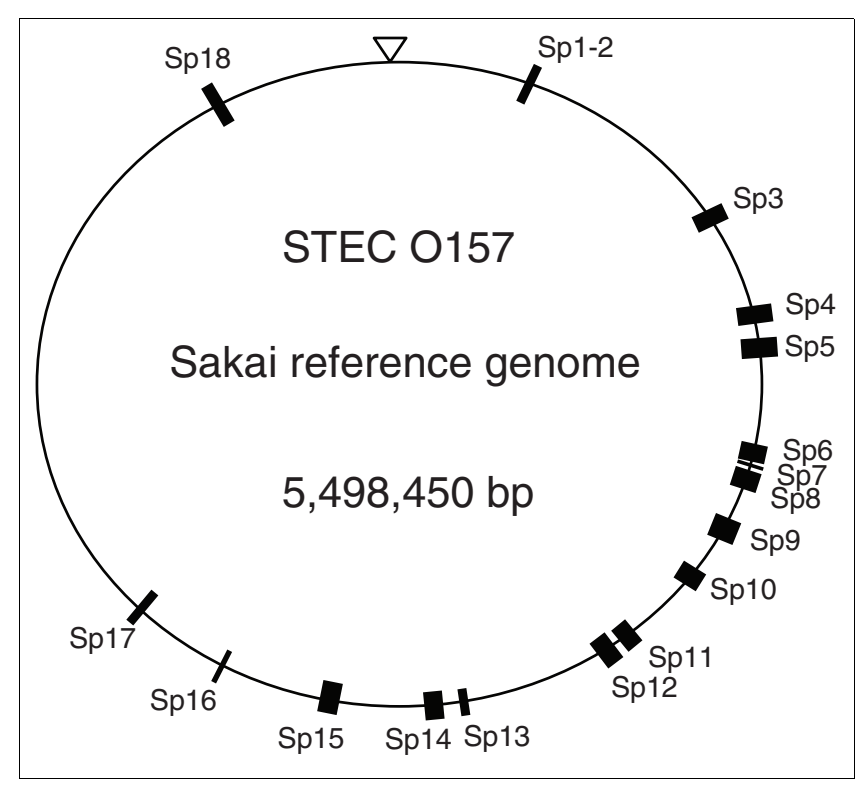

Figure I

Regions of the STEC OI57 genome (Sakai reference strain) targeted for polymorphism validation. Black rectangles represent known phage or phage remnant integrations (Sakai prophages; Sp I- 18, [4]) that were not queried for polymorphism validation. All other regions, totaling $87.8 \%$ of the genome, were included for polymorphism validation. The triangle points to nucleotide I of the Sakai genome sequence [GenBank:NC 002695]. one another. As a result, apparent polymorphisms may actually be differences between two or more highly similar sites in the genome rather than representing true variation at a single nucleotide locus. In addition, assay design in these highly repetitive sequences is impractical. Consequently, putative polymorphisms identified within these sites were not queried with validation assays in this study. Of the remaining 6,690 putative polymorphisms, 1,735 were identified via the STEC O157 DNA pool of human strains, and 4,955 were identified via the DNA pools of cattle strains.

Matrix-assisted laser desorption-ionization time-of-flight (MALDI-TOF) genotype validation assays were developed for 227 putative polymorphisms based on their minor allele frequencies in one or more of the STEC O157 DNA pools. The minor alleles of 169 polymorphisms were observed exclusively in one of the three DNA pools at a frequency of $15 \%$ or higher (human strain DNA pool $(n=18)$, bovine strain DNA pool, tir $255 \mathrm{~T}>\mathrm{A}$ T allele $(\mathrm{n}=81$ ), bovine strain DNA pool, tir $255 \mathrm{~T}>$ A A allele $(\mathrm{n}=70)$ ). Additionally, 58 polymorphisms were included where the minor allele was observed in both bovine DNA pools with a minor allele frequency of $10 \%$ or higher in one or both pools. MALDI-TOF genotyping of the 227 polymorphisms across 261 STEC O157 strains (Additional data file 1) indicated that 21 putative polymorphisms resided in duplicated genomic regions as a subset of STEC O157 strains yielded heterozygous genotypes. Another 28 putative polymorphisms proved either intractable for geno- typing or yielded monomorphic genotypes. Of 178 polymorphisms validated by MALDI-TOF genotyping, 139 reside in open reading frames with 86 predicted non-synonymous or premature stop codon allele variants. Additionally, 154 reside on the conserved genomic backbone of $E$. coli (see Additional data file 2 for supplementary polymorphism information).

\section{Identification of polymorphism-derived genotypes in STEC O I57 strains of human and cattle origin}

Concatenation of 178 polymorphism alleles for each of the STEC O157 strains genotyped in this study yielded 42 unique polymorphism-derived genotypes that are delineable with a minimal subset of 32 'tagging' polymorphisms (see Additional data files 3 and 4 for polymorphism-derived genotypes based on all 178 and the 32 tagging polymorphisms, respectively). A total of 34 of the polymorphism-derived genotypes were observed in STEC O157 strains of cattle origin, 16 were observed in strains of human origin, with 8 observed in strains of both human and cattle origin (Figure 2; Additional data file 1). Eight genotypes were observed exclusively in strains of human origin and 26 were observed exclusively in strains of bovine origin. Of particular interest, the same STEC O157 genotype (genotype 28) had the highest overall frequency in STEC O157 strains of human and bovine origin (Figure 2), indicating that STEC O157 of this genetic background may have an advantage in populating cattle and/or causing disease in humans.

\section{Phylogenetic analyses of STEC OI57 polymorphism- derived genotypes}

Neighbor-joining, parsimony, and maximum-likelihood trees were generated for the 42 polymorphism-derived genotypes using 178 polymorphism alleles, and the minimal set of 32 tagging polymorphism alleles. Both allele data sets yielded similar trees; however, bootstrap values were lower overall in trees generated with the minimal set of 32 tagging polymorphism alleles, as this set contained a reduced amount of phylogenetic information (Figure 3; see Additional data file 5 for a phylogenetic tree based on the 32 tagging polymorphism alleles). The trees were used to depict the genetic relatedness of STEC O157 strains of known host origin and tir 255T $>A$ allele status. The neighbor-joining tree in Figure 3 was constructed from 178 polymorphism alleles, and shows a monophyletic cluster of all 17 polymorphism-derived genotypes with the tir $255 \mathrm{~T}>\mathrm{A}$ A allele. Strains from 66 cattle originating from the US, Japan, Scotland, and Australia had the tir $255 \mathrm{~T}>\mathrm{A}$ A allele and one of the 17 polymorphism-derived genotypes. Additionally, the one STEC O157 strain of human origin included in this study that had the tir $255 \mathrm{~T}>\mathrm{A}$ A allele also had a genotype contained within the cluster (Additional data files 1, 3 and 4). The remaining 25 polymorphism-derived genotypes represent STEC O157 strains isolated from humans or cattle that all have the tir $255 \mathrm{~T}>\mathrm{A}$ T allele. These genotypes cluster together in subclades that are strongly supported by neighbor-joining, parsimony, and maximum-likelihood algorithms (Figure 3). Ninety-two percent of the human STEC 


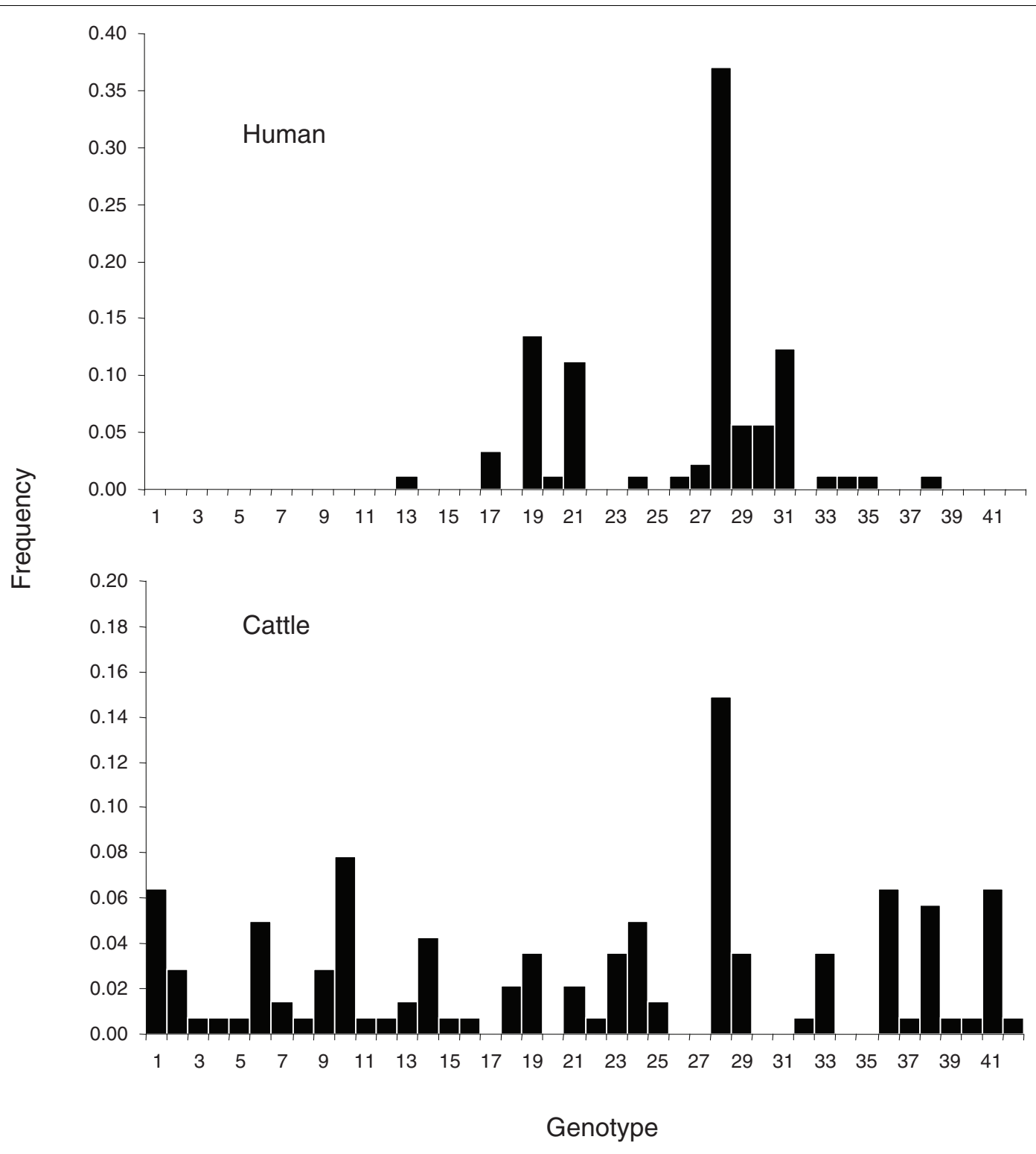

Figure 2

Frequencies of 42 polymorphism-derived genotypes in STEC OI57 strains of human and cattle origin.

O157 strains genotyped in this study placed within two subclades on the tree (Figure 3).

To determine the extent to which the polymorphism-derived genotypes can be used to distinguish STEC O157 genetic relatedness, a median-joining network was constructed from the 32 tagging polymorphism data set (Figure 4). Unlike the neighbor-joining, parsimony, and maximum-likelihood trees, which placed genotypes exclusively as outer taxonomic units, the median-joining network allowed for genotypes to be placed as either internal or outer nodes of the network. Nodes on linear, open, connecting lines on this network represent stepwise evolutionary descent. Nodes on circular, closed loops in the center of the network indicate that either convergent evolution or recombination occurred within some STEC O157 strains (Figure 4) [8]. Given that lateral-gene transfer is a fundamental component of STEC O157 biology and pathogenesis, and that a tri-allelic polymorphism was identified in this study (Additional data file 2; nucleotide position 3,506,470, Additional data files 3 and 4), either scenario is likely and both confound interpretations of genetic relatedness on the network, which assumes stepwise evolutionary descent. Consequently, the loops within the center of the network provide a natural barrier in determining genetic relatedness between STEC O157 genotypes (Figure 4). 


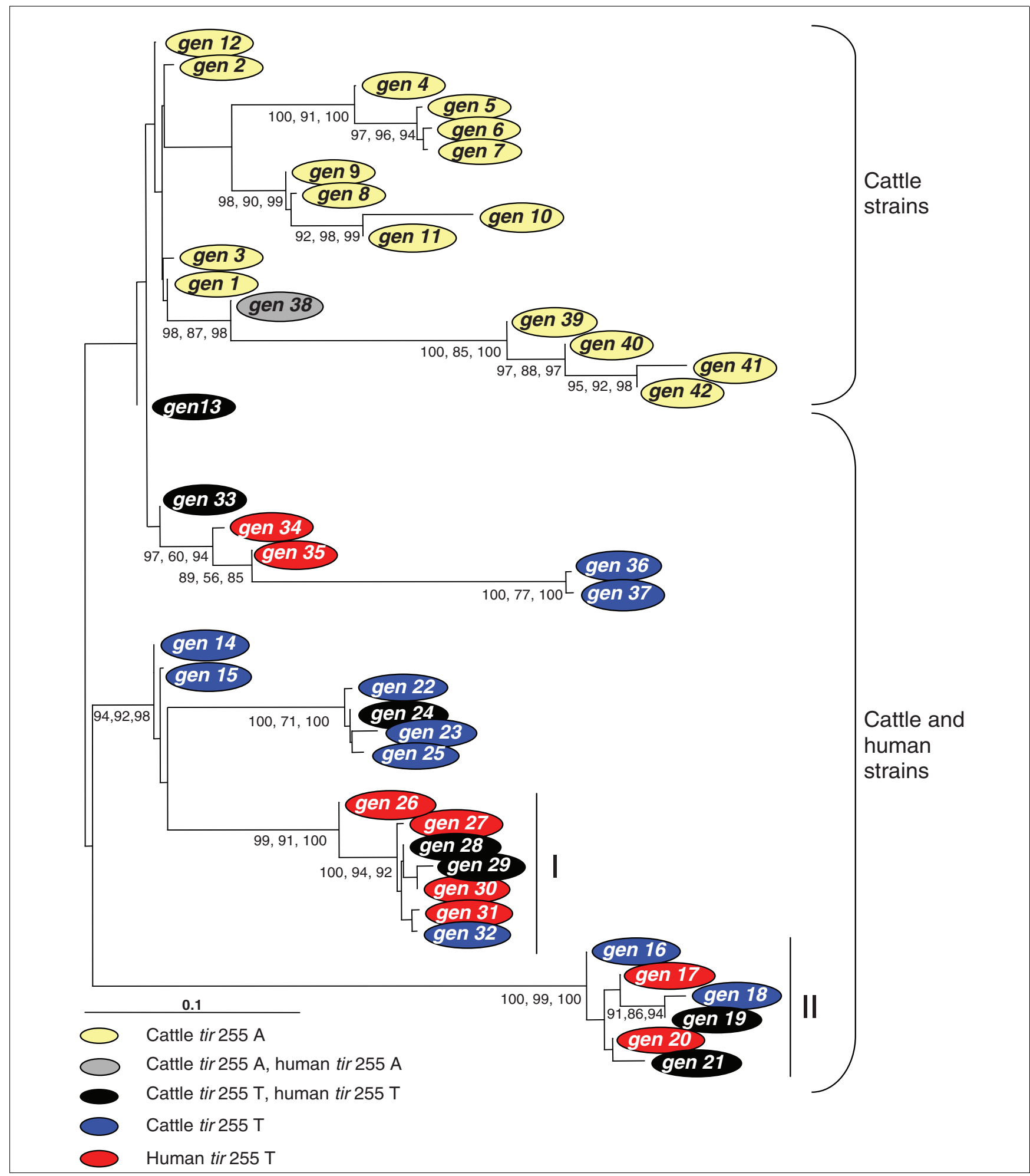

Figure 3

Neighbor-joining tree of full-length polymorphism-derived genotypes. The triplicate sets of numbers on the tree represent bootstrap values from neighbor-joining, parsimony, and maximum-likelihood algorithms, respectively. Outer taxonomic unit genotype numbers correspond with genotype sequences recorded in Additional data file 3. The outer taxonomic units are color coded by genotype for the tir $255 \mathrm{~T}>\mathrm{A}$ polymorphism and host origin. Roman numerals depict two subclades that account for $92 \%$ of the human STEC OI57 strains genotyped in this study. The scale bar represents substitutions per site. 


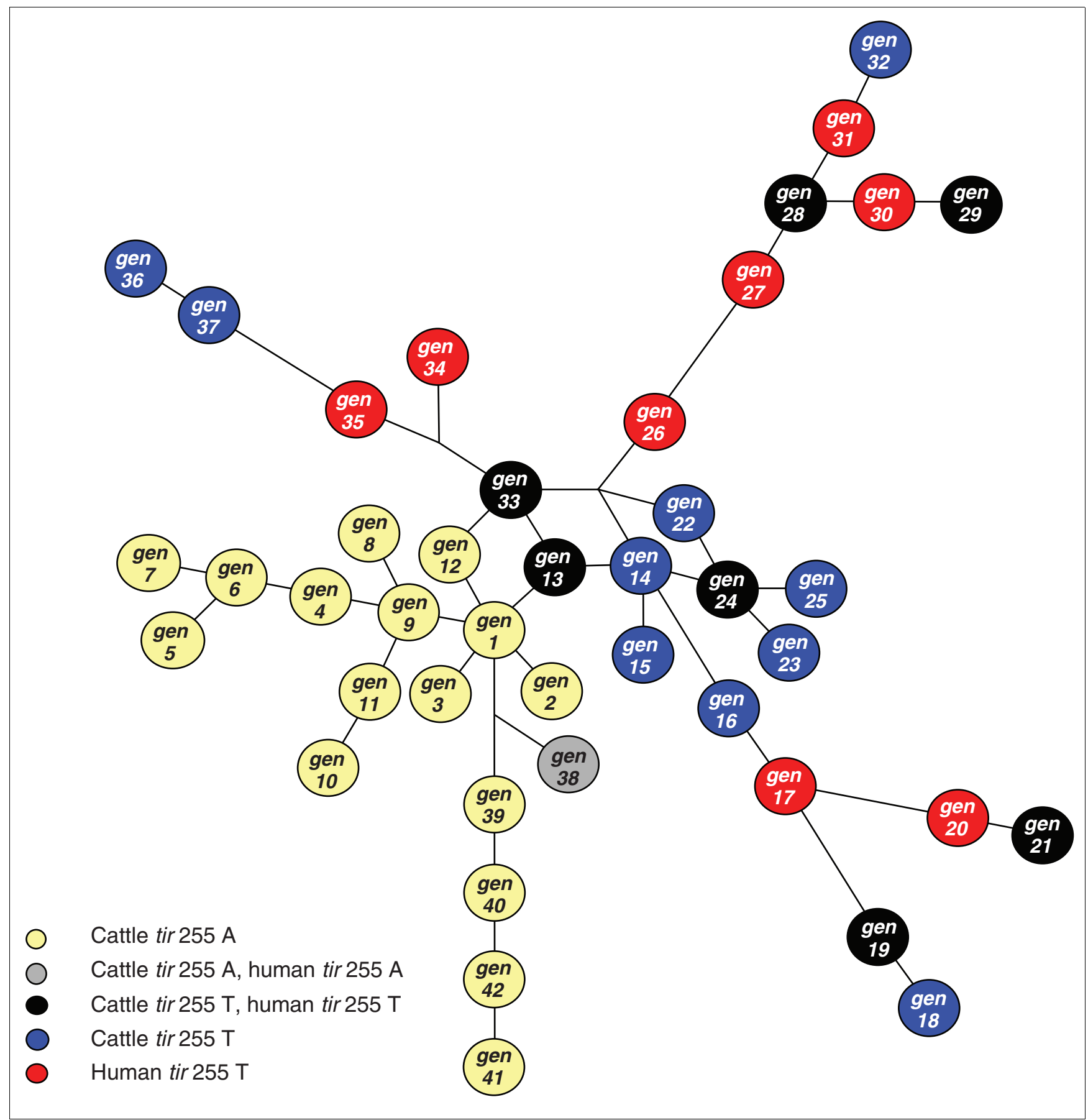

Figure 4

Median-joining network of polymorphism-derived genotypes tagged with a minimal set of 32 polymorphisms. Taxonomic unit genotype numbers correspond with genotype sequences recorded in Additional data file 4 and the units are color coded by genotype for the tir 255 T>A polymorphism and host origin.

\section{Comparison of PFGE and polymorphism-derived genotype diversity}

PFGE patterns and polymorphism-derived genotypes were compared between 227 epidemiologically unrelated STEC O157 strains using unambiguous PFGE patterns and polymorphism derived-genotypes (Additional data file 1). A con- servative standard was employed for distinguishing differing PFGE patterns, where only identical banding patterns were assigned to the same PFGE group. We observed 154 PFGE patterns and 42 polymorphism-derived genotypes between the strains, with multiple PFGE patterns observed on 24 genotypes (Figure 5; Additional data file 1). A total of 131 PFGE 
patterns and 18 polymorphism-derived genotypes manifested as singletons in this study (Additional data file 1). Of 23 PFGE patterns observed in more than one strain, 10 occurred with strains having different polymorphism-derived genotypes, with 3 PFGE patterns each manifesting in strains of markedly different genetic backgrounds (Figure 6). This result indicates that the polymorphism-derived genotypes described in this study have an immediate utility in distinguishing genetically distinct STEC O157 strains that appear identical by PFGE profile.

\section{Discussion}

GS FLX sequences are clonal in origin because they are ultimately derived from a single strand of DNA. Consequently, high- and low-frequency polymorphism alleles can be detected through GS FLX sequencing of pooled DNA libraries. We took advantage of this attribute by designing STEC O157 DNA pools that were sorted by host origin phenotype (cattle or human), and genotype for the tir $255 \mathrm{~T}>\mathrm{A}$ polymorphism. Because the tir $255 \mathrm{~T}>\mathrm{A}$ A allele is rarely observed in STEC O157 isolated from humans, STEC O157 DNAs of cattle origin could be separated into two pools, one representing a portion of STEC O157 diversity that appears primarily in cattle, and one representing a portion of STEC O157 diversity that may or may not appear in clinically ill humans. These two pools were complemented with the DNA pool of STEC O157 strains isolated from clinically ill humans. By selecting polymorphisms where the minor allele was observed at a relatively high frequency in either the STEC O157 DNA pool of human strains or at least one of the two cattle strain DNA pools, 42 polymorphism genotypes were identified that cover a large spectrum of STEC O157 diversity present in cattle, and a subset of genotypes that manifests in clinically ill humans.

While this study defined a sub-lineage of STEC O157 that is poorly represented in humans, a genetic mechanism for causing this host restriction is unknown. The tir 255T $>$ A polymorphism is a likely candidate as the translocated intimin receptor protein is part of the STEC O157 type-three secretion system and facilitates bacterium attachment to enterocyte cells within the colon and subsequent effacement [27]. Additionally, the $\mathrm{T}>\mathrm{A}$ substitution encodes a non-synonymous replacement of aspartate for glutamate in the translocated intimin receptor protein. However, the tir $255 \mathrm{~T}>\mathrm{A}$ polymorphism is not known to directly affect STEC O157 virulence in

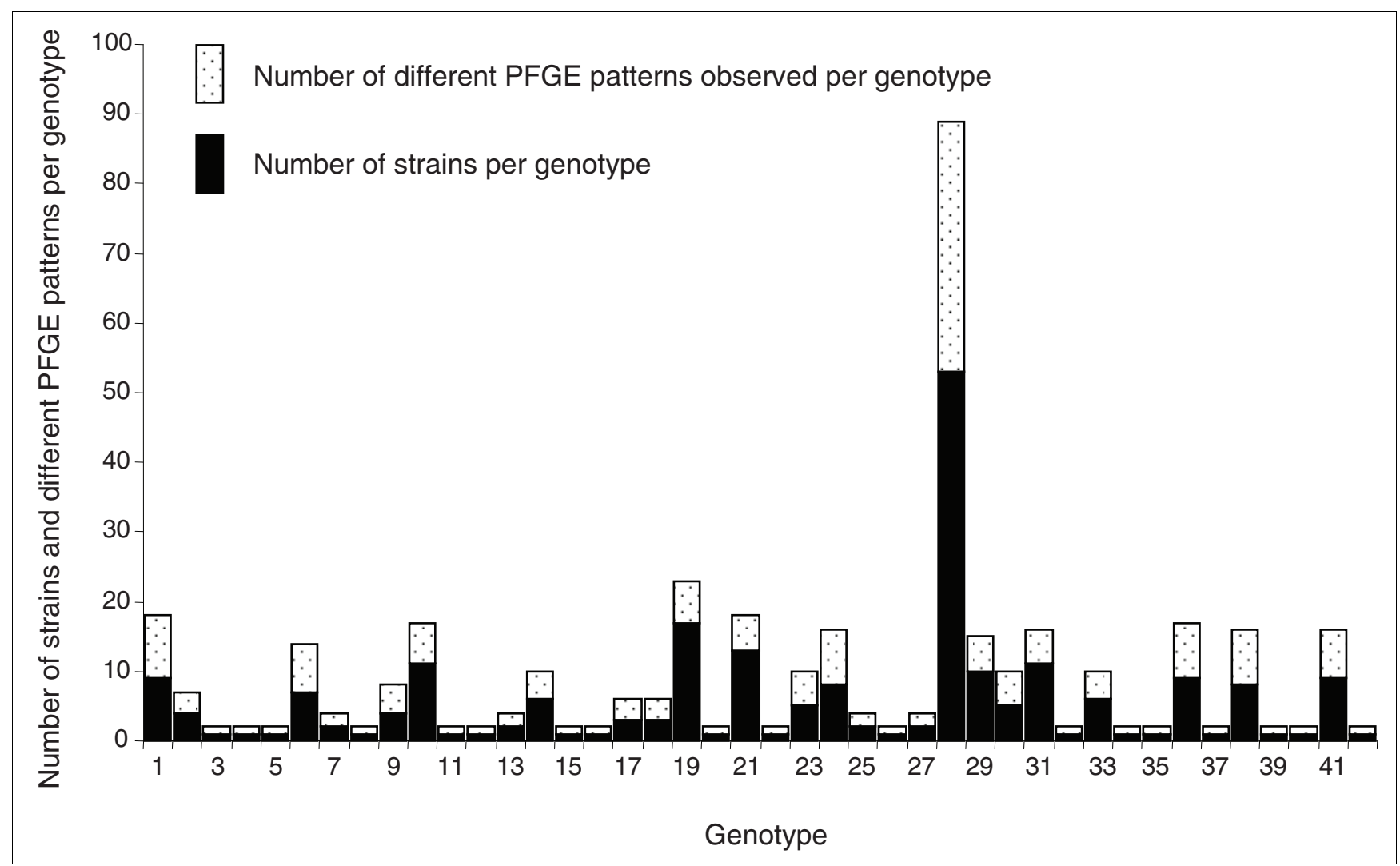

Figure 5

Number of strains and PFGE patterns per genotype. Each stacked bar represents a total of the number of strains per genotype and the number of different PFGE patterns observed per genotype. The black portion of the bars represents the number of strains per genotype. The white speckled portions of the bars represent the number of different PFGE patterns observed per genotype. 


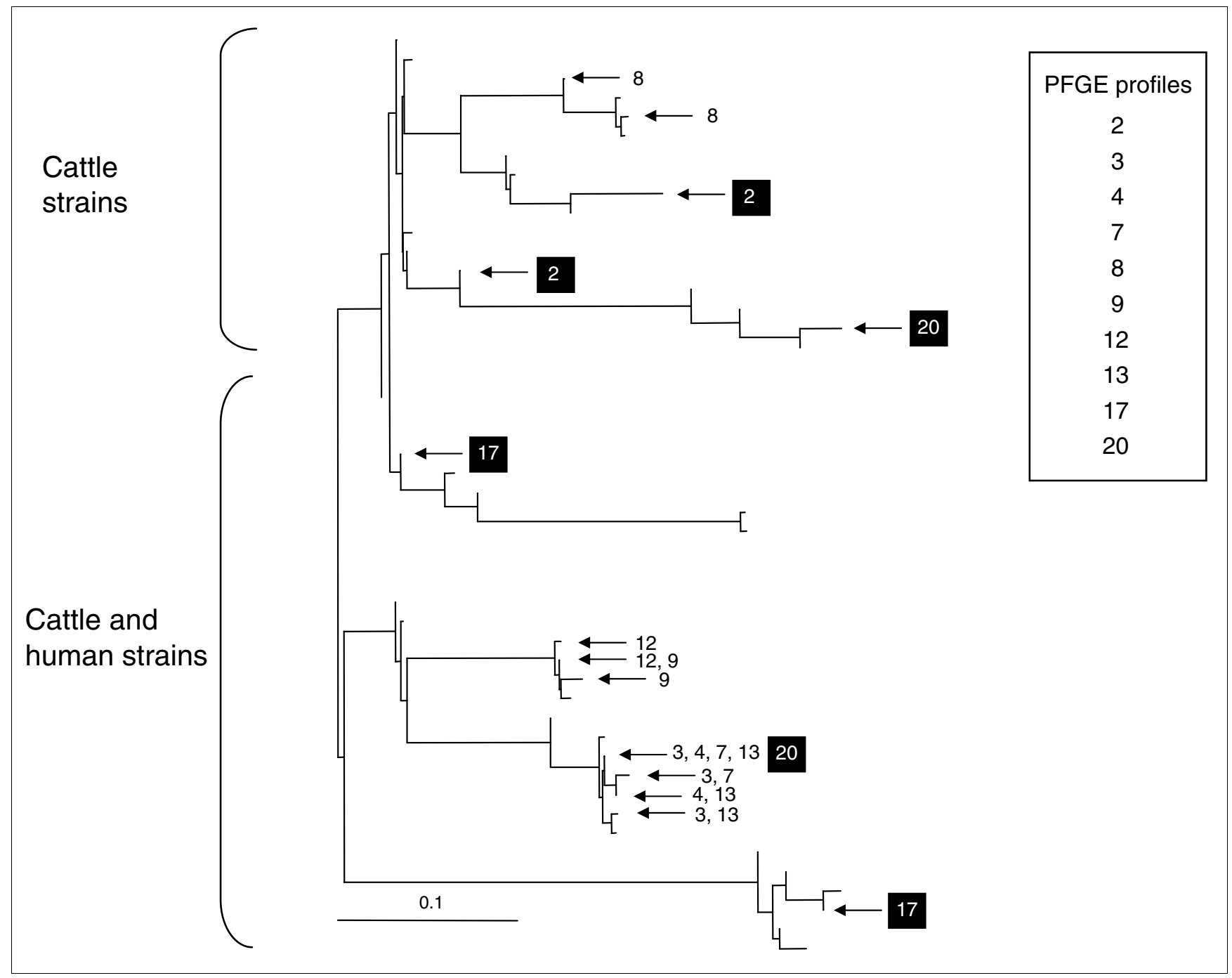

\section{Figure 6}

Neighbor-joining tree placement of ten PFGE profiles onto corresponding polymorphism-derived genotypes. Each of the ten profiles was observed with more than one STEC OI57 strain. The PFGE profile numbers match with those in Additional data file I. Identical PFGE profiles that occurred with distantly related STEC OI57 strains as determined with polymorphism-derived genotypes are highlighted in black.

humans [26], and this study identified 1,735 putative polymorphisms (outside of phage integration sites) with minor alleles exclusively observed in the human STEC O157 DNA pool. This finding complicates interpretations regarding a tir $255 \mathrm{~T}>\mathrm{A}$ polymorphism effect on human virulence. Regardless of knowing which alleles directly impact the ability of STEC O157 to cause human disease, an ability to track and identify variation linked with the tir $255 \mathrm{~T}>\mathrm{A}$ A allele is important, as one human strain in this study had the tir $255 \mathrm{~T}>\mathrm{A} A$ allele and a polymorphism-derived genotype that fell within the monophyletic clade typically found in cattle.

A majority of STEC O157-induced human disease sampled in this study was caused by strains that have followed one of two overarching lines of descent, as $92 \%$ of all human strain polymorphism-derived genotypes were placed within two large subclades (Figures 3 and 4; Additional data file 5). These two clades are separated from one another by both orthologous descent and probable recombination (Figure 4) and may be split out further into smaller clade sets [8]. It is likely that variation between the subclades, or variation among genotypes within a subclade, may associate with human virulence, as this has been previously demonstrated with the US spinach outbreak strain of 2006 [8], which had a higher rate of hospitalization and hemolytic uremic syndrome than other outbreak strains [28]. The US spinach outbreak strain was included in this study and has a polymorphism-derived genotype (genotype 21) that differs from all others, in that only it contains the minor alleles of two non-synonymous polymorphisms (Additional data files 2 and $3, \mathrm{~N}$-acetylglutamate synthase: alanine to serine (position $3,672,410$ ), and cytochrome c nitrite reductase: arginine to histidine (position 5,141,169)). 
These polymorphisms were not characterized in a previous study of STEC O157 polymorphism-derived genotypes and human virulence [8] as only four polymorphisms used in that study coincide with the 178 described here.

The polymorphisms validated in this study primarily reside in the conserved backbone of $E$. coli and some may be informative across Escherichia species. PFGE, the current gold standard for assessing STEC O157 genetic diversity [21], primarily detects insertions and/or deletions within genomic regions specific to STEC O157 [29]. Consequently, PFGE and the polymorphism-derived genotypes described in this study target different regions of the STEC O157 genome that do not share a common phylogeny. It is not surprising that PFGE diversity surpassed polymorphism-derived genotype diversity overall, given that PFGE patterns are known to change between subcultures of the same strain of STEC O157:H7 [30] and that plasmid migration within PFGE can be unpredictable [23]. Future studies should be conducted that compare STEC O157 diversity assessed with the polymorphism-derived genotypes and PFGE using outbreak samples. However, given that ten different PFGE patterns were each observed in two or more strains with different polymorphism genotypes, the 42 polymorphism-derived genotypes identified in this study have immediate potential to resolve genetically distinct STEC O157 strains comprising an outbreak investigation that may be indistinguishable by PFGE.

\section{Conclusions}

The method of pooling large numbers of phenotyped STEC O157 strain DNAs and subsequent high throughput 454 sequencing proved extremely efficient for the identification of variation within and between pooled populations, and resulted in the identification of 178 polymorphisms that collectively define 42 unique STEC O157 genotypes. The genotypes characterize genetic diversity and relatedness within STEC O157 strains of bovine origin, and a subset observed in human strains. We identified a minimal set of 32 polymorphisms that tag all 42 genotypes, and show that this set can detect genetically diverse STEC O157 strains that are indistinguishable by PFGE.

\section{Materials and methods Bacterial strains}

STEC O157 strains of bovine origin $(n=102)$ that varied by source, and epidemiologically unrelated human clinical STEC O157 strains $(\mathrm{n}=91)$ were used for polymorphism discovery (Additional data file 1) [4,31-37]. Each strain was characterized as STEC O157 by an enzyme-linked immunosorbent assay using an O157 monoclonal antibody and multiplex PCR for stx1, stx2, eae, hlyA, $r f b_{\mathrm{O}_{157}}$ and $\mathrm{fliC}_{\mathrm{H}_{7}}$ [38-41]. Additionally, each strain was genotyped for a polymorphism residing within the translocated intimin receptor gene (tir $255 \mathrm{~T}>\mathrm{A}$ ) [26]. A total of 261 STEC O157 strains, 164 isolated from cattle and 97 isolated from human were targeted for genotyping of: tir $255 \mathrm{~T}>\mathrm{A}$; 178 polymorphisms identified in this study; and PFGE (Additional data file 1).

\section{DNA isolation}

Genomic DNA was extracted from STEC O157 strains using Qiagen Genomic-tip 100/G columns (Valencia, CA, USA) and a modified manufacturer's protocol. Following overnight growth in $5 \mathrm{ml}$ of Luria broth, bacteria were pelleted by centrifugation at 5,000 $\times \mathrm{g}$ for 15 minutes, re-suspended in Qiagen buffer B1 containing RNase A ( $0.2 \mathrm{mg} / \mathrm{ml})$, and vortexed per the manufacturer's instructions. Importantly, the samples were then incubated at $70^{\circ} \mathrm{C}$ for 10 minutes, vortexed, and equilibrated at $37^{\circ} \mathrm{C}$ (failure to include the $70^{\circ} \mathrm{C}$ step frequently resulted in the columns becoming plugged and/or a significant decrease in DNA yield). Following the addition of $80 \mu \mathrm{l}$ lysozyme (100 $\mathrm{mg} / \mathrm{ml}$ ), $100 \mu \mathrm{l}$ proteinase K (Qiagen), and a $37^{\circ} \mathrm{C}$ incubation for 30 minutes, the DNAs were extracted and air dried per the manufacturer's protocol. Purified DNAs were suspended in $500 \mu \mathrm{TE}$ (10 mM Tris $\mathrm{pH}$ 8.o, $0.1 \mathrm{mM}$ EDTA) and incubated for 2 hours at $50^{\circ} \mathrm{C}$, followed by an overnight incubation at room temperature with gentle mixing. Strain DNA preparations were assessed by $260 \mathrm{~nm} /$ $280 \mathrm{~nm}$ absorptions, which were determined with a NanoDrop Technologies ND-100o spectrophotometer (Wilmington, DE, USA), and by gel electrophoresis.

\section{STEC OI57 DNA pools, GS FLX sequencing, and polymorphism identification}

Three STEC O157 DNA pools were created for GS FLX sequencing and polymorphism discovery. One consisted of DNAs from 51 STEC O157 strains (3 $\mu \mathrm{g} /$ strain), all of cattle origin and all with the tir $255 \mathrm{~T}>\mathrm{A}$ A allele. Another consisted of DNAs from 51 STEC O157 strains ( $3 \mu \mathrm{g} /$ strain), all of cattle origin and all with the tir $255 \mathrm{~T}>\mathrm{A} \mathrm{T}$ allele. Another consisted of DNAs from 91 STEC O157 strains ( $3 \mu \mathrm{g} /$ strain) originating from clinically ill humans, all with the tir $255 \mathrm{~T}>\mathrm{A} \mathrm{T}$ allele. Genomic libraries were prepared from each of the three DNA pools for Roche 454 GS FLX shot-gun sequencing according to the manufacturer's protocol (Nutley, NJ, USA). A total of 11 emulsion-based PCRs and sequencing runs were performed, three for the DNA pool of cattle origin, tir 255T $>$ A A allele, three for the DNA pool of cattle origin, tir $255 \mathrm{~T}>\mathrm{A} \mathrm{T}$ allele, and five for the DNA pool of human origin. SNPs were mapped to a reference sequence of STEC O157 (Sakai strain) and identified with Roche GS Reference Mapper Software (version 1.1.03).

\section{Polymorphism genotyping}

A file containing all targeted polymorphisms was prepared for assay design and multiplexing by MassARRAY ${ }^{\circledR}$ assay design software as recommended by the manufacturer (Sequenom, Inc., San Diego, CA, USA). A target of maximum 36 and minimum 21 polymorphisms per multiplex was set for design, with default settings for all other parameters. Seven multiplexes containing 225 polymorphisms were designed (aver- 
age 32 polymorphisms per multiplex, range 21 to 36). Assays were performed using iPLEX Gold ${ }^{\circledR}$ chemistry on a MassAR$\mathrm{RAY}^{\circledR}$ genotyping system as recommended by the manufacturer (Sequenom Inc.). Genotypes designated as high confidence by the Genotyper ${ }^{\circledR}$ software were accepted as correct; those with lower confidence (marked 'aggressive' in the software) were manually inspected. Replicate iPLEX assays and/or Sanger sequencing were used to verify genotypes.

\section{Polymorphism-derived genotype analyses}

The alleles of 178 polymorphisms were concatenated by physical order along the STEC O157 genome for 261 STEC O157 strains and aligned using Clustal $X$ (version 1.83) [42]. Redundant polymorphism-derived genotypes were identified using TreePuzzle (version 5.2) [43,44], and removed from Clustal X alignments. Neighbor-joining and parsimony phylogenetic trees were generated using a collection of software programs in PHYLIP (version 3.65, Consense, DnaDist, DnaPars, Neighbor, Retree, Seqboot) [45]. To construct a neighbor-joining tree, a distance matrix was first produced in DnaDist using an F84 distance model of substitution and a transition/transversion ratio of 2. The output of DnaDist was used to construct a neighbor-joining tree in Neighbor, which was mid-point rooted using Retree. Neighbor-joining bootstraps (1,00o) were determined with Seqboot, DnaDist, Neighbor, and Consense. A parsimony tree with 1,00o bootstraps was generated with Seqboot, DnaPars (best tree thorough search) and Consense. Maximum-likelihood trees were generated in Tree-Puzzle (version 5.2) with 10,000 puzzling steps and an HKY model of substitution. Neighbor-joining, parsimony, and maximum-likelihood trees were all viewed in TreeView (version 1.6.6) [46].

Haploview v 4.1 [47] was used to identify a minimal set of polymorphisms (tagging polymorphisms) that distinguish each of the unique polymorphism-derived genotypes observed in this study. All 178 polymorphism genotypes were used to infer STEC O157 haplotypes in Haploview at a haplotype frequency threshold of o\% or higher. Neighbor-joining, parsimony, and maximum-likelihood trees were generated from concatenated tagging polymorphism genotypes using model assumptions identical to those used for the full genotype data sets. Additionally, a median-joining network was constructed in Network (version 4.5.0.2) [48] for the concatenated tagging polymorphism genotypes.

\section{Pulsed field gel electrophoresis}

The standardized PFGE method [49] was performed on 261 STEC O157 strains that were also targeted for SNP genotyping (Additional data file 1). Gel images were analyzed using Bionumerics (Applied Maths, Sint-Martens-Latem, Belgium), and banding patterns were clustered using an unweighted pairgroup method with arithmetic mean algorithm and a bandbased Dice coefficient. Default tolerance settings were used. No restriction enzymes additional to $X b a I$ were used. Strains were assigned to the same PFGE group only if XbaI banding patterns were indistinguishable.

\section{Abbreviations}

MALDI-TOF: matrix-assisted laser desorption-ionization time-of-flight; PFGE: pulsed-field gel electrophoresis; SNP: single nucleotide polymorphism; STEC O157: Shiga toxincontaining Escherichia coli O157:H7.

\section{Authors' contributions}

MLC conducted experimental design and data generation, analyzed 454 GS FLX and MALDI-TOF results, performed phylogenetic analyses, and wrote the manuscript. JEK conceived the project, characterized STEC O157 strains, and participated in 454 GS FLX sequencing design. TPLS participated in experimental design and STEC O157 DNA purification, conducted 454 GS FLX library construction and sequencing, and MALDI-TOF genotyping. LMD characterized STEC O157 strains, performed and analyzed PFGE, and participated in STEC O157 DNA purification. TGM participated in STEC O157 DNA purification and 454 GS FLX library construction and sequencing. REM characterized epidemiologically related STEC O157 strains. MAD characterized an international collection of STEC O157 strains and provided PFGE results. JLB participated in experimental design, conducted STEC O157 characterizations, culture, and DNA isolations, and analyzed 454 GS FLX and MALDI-TOF results.

\section{Additional data files}

The following additional data are available with the online version of this paper: a table of STEC O157 strains used in this study with their corresponding PFGE patterns and polymorphism-derived genotypes (Additional data file 1); a table of nucleotide polymorphism allele frequencies in STEC O157 strains of bovine and human origin (Additional data file 2); a table of STEC O157 genotypes defined by 178 nucleotide polymorphisms (Additional data file 3); a table of STEC O157 genotypes defined by a minimal set of 32 nucleotide polymorphisms (Additional data file 4); a figure showing neighborjoining tree of polymorphism-derived genotypes tagged with a minimal set of 32 polymorphisms (Additional data file 5).

\section{Acknowledgements}

We thank Renee Godtel, Bob Lee, Sandy Fryda-Bradley, Gennie SchullerChavez, Kevin Tennill, Linda Flathman, Ron Mlejnek, Scott Schroetlin, and Casey Trambly for outstanding technical support for this project; Dr Thomas E Besser for his generous donations of STEC OI 57 strains; Dr Michael P Heaton for helpful discussions on experimental design; Jim Wray, Phil Anderson, and Randy Bradley for computer support; Joan Rosch for secretarial support, and Drs Jeffrey Gawronski and David Benson for reviewing the manuscript. This work was supported in part by The Beef Checkoff (MLC JEK, JLB) and the Agricultural Research Service (MLC, JEK, TPLS, LMD, TGM, REM, JLB). The use of product and company names is necessary to accurately report the methods and results; however, the USDA neither guarantees nor warrants the standard of the products, and the use of 
names by the USDA implies no approval of the product to the exclusion of others that may also be suitable.

\section{References}

I. Griffin PM, Tauxe RV: The epidemiology of infections caused by Escherichia coli OI57:H7, other enterohemorrhagic E. coli, and the associated hemolytic uremic syndrome. Epidemiol Rev 1991, 13:60-98.

2. Mead PS, Slutsker L, Dietz V, McCaig LF, Bresee JS, Shapiro C, Griffin PM, Tauxe RV: Food-related illness and death in the United States. Emerg Infect Dis 1999, 5:607-625.

3. Whittam TS, Wolfe ML, Wachsmuth IK, Orskov F, Orskov I, Wilson RA: Clonal relationships among Escherichia coli strains that cause hemorrhagic colitis and infantile diarrhea. Infect Immun 1993, 61:1619-1629.

4. Hayashi T, Makino K, Ohnishi M, Kurokawa K, Ishii K, Yokoyama K, Han CG, Ohtsubo E, Nakayama K, Murata T, Tanaka M, Tobe T, lida T, Takami H, Honda T, Sasakawa C, Ogasawara N, Yasunaga T, Kuhara S, Shiba T, Hattori M, Shinagawa H: Complete genome sequence of enterohemorrhagic Escherichia coli O157:H7 and genomic comparison with a laboratory strain K-I2. DNA Res 200I, 8: II-22.

5. Perna NT, Plunkett G 3rd, Burland V, Mau B, Glasner JD, Rose DJ, Mayhew GF, Evans PS, Gregor J, Kirkpatrick HA, Pósfai G, Hackett J, Klink S, Boutin A, Shao Y, Miller L, Grotbeck EJ, Davis NW, Lim A, Dimalanta ET, Potamousis KD, Apodaca J, Anantharaman TS, Lin J, Yen G, Schwartz DC, Welch RA, Blattner FR: Genome sequence of enterohaemorrhagic Escherichia coli O 157:H7. Nature 200I, 409:529-533.

6. Feng P, Lampel KA, Karch H, Whittam TS: Genotypic and phenotypic changes in the emergence of Escherichia coli O157:H7. J Infect Dis 1998, I77: 1750-1753.

7. Feng PCH, Monday SR, Lacher DW, Allison L, Siitonen A, Keys C, Eklund M, Nagano H, Karch H, Keen J, Whittam TS: Genetic diversity among clonal lineages within Escherichia coli OI57:H7 stepwise evolutionary model. Emerg Infect Dis 2007, 13:1701-1706.

8. Manning SD, Motiwala AS, Springman AC, Qi W, Lacher DW, Ouellette LM, Mladonicky JM, Somsel P, Rudrik JT, Dietrich SE, Zhang W, Swaminathan B, Alland D, Whittam TS: Variation in virulence among clades of Escherichia coli O I 57:H7 associated with disease outbreaks. Proc Natl Acad Sci USA 2008, 105:4868-4873.

9. Afset JE, Anderssen E, Bruant G, Harel J, Wieler LH, Bergh K: Phylogenetic backgrounds and virulence profiles of atypical enteropathogenic Escherichia coli strains from a case-control study using multilocus sequence typing and DNA microarray analysis. J Clin Microbiol 2008, 46:2280-2290.

10. Kim J, Nietfeldt J, Benson AK: Octamer-based genome scanning distinguishes a unique subpopulation of Escherichia coli O157:H7 strains in cattle. Proc Natl Acad Sci USA 1999, 96:13288-13293.

II. Ohnishi M, Terajima J, Kurokawa K, Nakayama K, Murata T, Tamura K, Ogura Y, Watanabe H, Hayashi T: Genomic diversity of enterohemorrhagic Escherichia coli 0157 revealed by whole genome PCR scanning. Proc Natl Acad Sci USA 2002, 99: $17043-17048$.

12. Ratnam S, March SB, Ahmed R, Bezanson GS, Kasatiya S: Characterization of Escherichia coli serotype O157:H7. J Clin Microbiol 1988, 26:2006-2012.

13. Ahmed R, Bopp C, Borczyk A, Kasatiya S: Phage-typing scheme for Escherichia coli O1 57:H7. J Infect Dis 1987, 155:806-809.

14. Keys C, Kemper S, Keim P: Highly diverse variable number tandem repeat loci in the $\mathrm{E}$. coli O 1 57:H7 and 055:H7 genomes for high-resolution molecular typing. J Appl Microbiol 2005, 98:928-940.

15. Jackson SA, Mammel MK, Patel IR, Mays T, Albert TJ, LeClerc JE, Cebula TA: Interrogating genomic diversity of $E$. coli OI 57:H7 using DNA tiling arrays. Forensic Sci Int 2007, 168:183-199.

16. Zhang W, Qi W, Albert TJ, Motiwala AS, Alland D, Hyytia-Trees EK, Ribot EM, Fields PI, Whittam TS, Swaminathan B: Probing genomic diversity and evolution of Escherichia coli 0157 by single nucleotide polymorphisms. Genome Res 2006, 16:757-767.

17. Shaikh N, Tarr PI: Escherichia coli O157:H7 Shiga toxin-encoding bacteriophages: integrations, excisions, truncations, and evolutionary implications. J Bacteriol 2003, 185:3596-3605.
18. Shaikh N, Holt NJ, Johnson JR, Tarr PI: Fim operon variation in the emergence of Enterohemorrhagic Escherichia coli: an evolutionary and functional analysis. FEMS Microbiol Lett 2007, 273:58-63.

19. Bohm $\mathrm{H}$, Karch $\mathrm{H}$ : DNA fingerprinting of Escherichia coli O 157:H7 strains by pulsed-field gel electrophoresis. J Clin Microbiol 1992, 30:2169-2I72.

20. Barrett TJ, Lior H, Green JH, Khakhria R, Wells JG, Bell BP, Greene $K D$, Lewis ], Griffin PM: Laboratory investigation of a multistate food-borne outbreak of Escherichia coli O157:H7 by using pulsed-field gel electrophoresis and phage typing. J Clin Microbiol 1994, 32:3013-3017.

21. Swaminathan B, Barrett TJ, Hunter SB, Tauxe RV, PulseNetTaskForce: PulseNet: the molecular subtyping network for foodborne bacterial disease surveillance, United States. Emerg Infect Dis 200I, 7:382-389.

22. Gerner-Smidt $P$, Hise K, Kincaid J, Hunter S, Rolando S, Hyytia-Trees E, Ribot EM, Swaminathan B, PulseNetTaskForce: PulseNet USA: a five-year update. Foodborne Pathog Dis 2006, 3:9-19.

23. Barrett T], Gerner-Smidt P, Swaminathan B: Interpretation of pulsed-field gel electrophoresis patterns in foodborne disease investigations and surveillance. Foodborne Pathog Dis 2006, 3:20-31.

24. Davis MA, Hancock DD, Besser TE, Call DR: Evaluation of pulsedfield gel electrophoresis as a tool for determining the degree of genetic relatedness between strains of Escherichia coli O157:H7. J Clin Microbiol 2003, 41:1843-1849.

25. Woese CR: Bacterial Evolution. Microbiol Rev 1987, 5 I:22 I-272.

26. Bono JL, Keen JE, Clawson ML, Durso LM, Heaton MP, Laegreid WW: Association of Escherichia coli O157:H7 tir polymorphisms with human infection. BMC Infect Dis 2007, 7:98.

27. Kenny B, DeVinney R, Stein M, Reinscheid DJ, Frey EA, Finlay BB: Enteropathogenic $E$. coli (EPEC) transfers its receptor for intimate adherence into mammalian cells. Cell 1997, 9I:5 II-520.

28. CDC: Ongoing multistate outbreak of Escherichia coli: serotype 0157:H7 infections associated with consumption of fresh spinach - United States, September 2006. MMWR Morb Mortal Wkly Rep 2006, 55: 1045-1046.

29. Kudva IT, Evans PS, Perna NT, Barrett T], Ausubel FM, Blattner FR, Calderwood SB: Strains of Escherichia coli O 157:H7 differ primarily by insertions or deletions, not single-nucleotide polymorphisms. J Bacteriol 2002, 184: 1873-1879.

30. Iguchi A, Osawa R, Kawano J, Shimizu A, Terajima J, Watanabe H: Effects of repeated subculturing and prolonged storage at room temperature of enterohemorrhagic Escherichia coli 0 157:H7 on pulse-field gel electrophoresis profiles. J Clin Microbiol 2002, 40:3079-308I.

31. Elder RO, Keen JE, Siragusa GR, Barkocy-Gallagher GA, Koohmaraie $M$, Laegreid WW: Correlation of enterohemorrhagic Escherichia coli 0157 prevalence in feces, hides, and carcasses of beef cattle during processing. Proc Natl Acad Sci USA 2000, 97:2999-3003.

32. Bono JL, Keen JE, Miller LC, Fox JM, Chitko-McKown CG, Heaton MP, Laegreid WW: Evaluation of a real-time PCR kit for detecting Escherichia coli 0157 in bovine fecal samples. Appl Environ Microbiol 2004, 70:1855-1857.

33. Keen JE, Wittum TE, Dunn JR, Bono JL, Durso LM: Shiga-toxigenic Escherichia coli 0157 in agricultural fair livestock, United States. Emerg Infect Dis 2006, I 2:780-786.

34. Whittam TS: Evolution of Escherichia coli O 157:H7 and other Shiga toxin-producing E. coli strains. In O157 Escherichia coli: $\mathrm{H7}$ and Other Shiga Toxin-producing E. coli Strains Edited by: Kaper JB, O'Brien AD. Washington, DC: ASM Press; 1998:195-209.

35. National Food Safety and Toxicology Center, Michigan State University: A Reference Center to Facilitate the Study of Shiga Toxin-producing Escherichia coli [http://www.shiga tox.net/cgi-bin/deca]

36. Davis MA, Hancock DD, Besser TE, Rice DH, Hovde CJ, Digiacomo R, Samadpour M, Call DR: Correlation between geographic distance and genetic similarity in an international collection of bovine faecal Escherichia coli O I 57:H7 isolates. Epidemiol Infect 2003, I 3 I:923-930.

37. Cooley M, Carychao D, Crawford-Miksza L, Jay MT, Myers C, Rose C, Keys C, Farrar J, Mandrell RE: Incidence and tracking of Escherichia coli O157:H7 in a major produce production region in California. PLOS ONE 2007, 2: el 159.

38. Gannon VPJ, D'Souza S, Graham T, King RK, Rahn K, Read S: Use of 
the flagellar $\mathbf{H 7}$ gene as a target in multiplex PCR assays and improved specificity in identification of enterohemorrhagic Escherichia coli strains. J Clin Microbiol 1997, 35:656-662.

39. He Y, Keen JE, Westerman RB, Littledike ET, Kwang J: Monoclonal antibodies for detection of the $\mathrm{H7}$ antigen of Escherichia coli. Appl Environ Microbiol 1996, 62:3325-3332.

40. Paton AW, Paton JC: Detection and characterization of Shiga toxigenic Escherichia coli by using multiplex PCR assays for stx I, stx2, eaeA, enterohemorrhagic E. coli hlyA, rfbO I I I, and rfbO I 57. J Clin Microbiol 1998, 36:598-602.

4I. Westerman RB, He Y, Keen JE, Littledike ET, Kwang J: Production and characterization of monoclonal antibodies specific for the lipopolysaccharide of Escherichia coli O I57. J Clin Microbiol 1997, 35:679-684.

42. Thompson JD, Gibson TJ, Plewniak F, Jeanmougin F, Higgins DG: The Clustal $X$ windows interface: flexible strategies for multiple sequence alignment aided by quality analysis tools. Nucleic Acids Res 1997, 25:4876-4882.

43. Schmidt HA, Strimmer K, Vingron M, von Haeseler A: TREE-PUZZLE: maximum likelihood phylogenetic analysis using quartets and parallel computing. Bioinformatics 2002, 18:502-504.

44. Strimmer K, von Haeseler A: Quartet puzzling: A quartet maximum likelihood method for reconstructing tree topologies. Mol Biol Evol 1996, I3:964-969.

45. PHYLIP (Phylogeny Inference Package) version 3.6 [http:// evolution.genetics.washington.edu/phylip.html]

46. Page RDM: TREEVIEW: An application to display phylogenetic trees on personal computers. Comput Appl Biosci 1996 , I 2:357-358.

47. Barrett JC, Fry B, Maller J, Daly MJ: Haploview: analysis and visualization of LD and haplotype maps. Bioinformatics 2005, 2I:263-265.

48. Bandelt HJ, Forster $P$, Rohl A: Median-joining networks for inferring intraspecific phylogenies. Mol Biol Evol 1999, 16:37-48.

49. Ribot EM, Fair MA, Gautom R, Cameron DN, Hunter SB, Swaminathan B, Barrett TJ: Standardization of pulsed-field gel electrophoresis protocols for the subtyping of Escherichia coli O I57:H7, Salmonella, and Shigella for PulseNet. Foodborne Pathog Dis 2006, 3:59-67. 